\title{
A PRÁTICA NA VIGILÂNCIA EPIDEMIOLÓGICA : ENTRE O GERAL E O ESPECÍFICO
}

\author{
THE EPIDEMIOLOGICAL SURVEILLANCE PRACTICE: BETWEEN THE GENERAL AND THE \\ SPECIFIC
}

\author{
LA PRÁCTICA PARA LA VIGILANCIA EPIDEMIOLÓGICA : ENTRE LO GENERAL YLO \\ ESPECÍFICO
}

\author{
Tereza Cristina Scatena Villa ${ }^{1}$ \\ Maria Cecilia Puntel de Almeida ${ }^{1}$ \\ Pedro Fredemir Palha ${ }^{1}$ \\ Jordana Nogueira Muniz ${ }^{2}$ \\ Roxana Izabel Cardoso Gonzales ${ }^{3}$ \\ José Martins Pinto Neto ${ }^{4}$ \\ Silvia Helena Vendramini ${ }^{5}$
}

RESUMO: O estudo analisa as perspectivas de organização da prática de vigilância epidemiológica(VE) em duas possibilidades organizacionais: "conjunto indiferenciado de práticas e conjunto singular das práticas no sistema local de saúde. Essas duas posições são descritas em traços gerais e discutidas sob a ótica dos profissionais de saúde do nivel local e da VE. Parte do pressuposto que a descentralização deve vir acompanhada da discussão de formas de organização do trabalho entre as equipes envolvidas. O eixo norteador são os conceitos de núcleo e campo de competência e responsabilidade. A população do estudo: 17 profissionais, 11 enfermeiras e 6 gestores/gerentes da SMS-RP. Através de uma abordagem qualitativa utilizou-se fontes documentais e entrevistas semi-estruturadas, através do método de análise de conteúdo, modalidade temática. O tema "A prática da Vigilância Epidemiológica: entre o geral e o específico" foi formado através de núcleos de sentido: conhecimento geral e específico e ações de caráter coletivo.

PALAVRAS-CHAVE: vigilância epidemiológica, descentralização, serviços de saúde

ABSTRACT: This study analyses the perspectives of the organization of epidemiological surveillance (ES) in two kinds of organizations. The first is a set of undifferentiated practices; the second is a set of specific practices carried out in the local health system of Ribeirão Preto, São Paulo Federal State. Both organizations are described in general terms and discussed according to the view of local health professionals and professionals of the epidemiological surveillance. This study presupposes that the decentralization should be supported by discussions regarding forms of organization among the groups involved in it. The guiding concepts of this research are nuclei and field of competence and responsibility. Seventeen professionals were the subjects of this study. Eleven participants were nurses and six were managers of the municipal health department of Ribeirão Preto. Data was obtained from documents and semi-structured interviews, which were analyzed through the method of content analysis, theme modality. The theme "the epidemiological surveillance practice: between the general and the specific" was created according to the following signification nuclei: specific and general knowledge, and collective actions. KEYWORDS: epidemiological surveillance, decentralization, health services

RESUMEN: El estudio analiza las perspectivas de organización para una práctica de vigilancia epidemiológica (VE) en dos posibilidades organizacionales: "conjunto indiferenciado de prácticas y conjunto singular de las prácticas en el sistema local de salud." Esas dos posturas están descritas en rasgos generales y están discutidas bajo la óptica de los profesionales de la salud del nivel local y de la VE. Se parte del presupuesto de que la descentralización debe estar acompañada de una discusión de las formas de organización del trabajo entre los equipos involucrados. El eje norteador son los conceptos de núcleo y campo de competencia y responsabilidad. La población del estudio: 17 profesionales, 11 enfermeras y 6 gestores/ gerentes de la SMS-RP. A través de un abordaje calitativo se han utilizado fuentes documentales y entrevistas semiestructuradas, mediante el método de análisis de contenido, la modalidad temática. El tema "La práctica de la Vigilancia Epidemiológica: entre lo general y lo específico" fue formado a través de núcleos de sentido: conocimiento general y especifico y acciones de carácter colectivo.

PALABRAS CLAVE: vigilancia epidemiológica, descentralización, organización de servicios de salud

Recebido em 22/04/2001

Aprovado em 26/06/2002

\footnotetext{
${ }^{1}$ Enfermeiros. Professores do Departamento de Enfermagem Materno Infantil e Saúde Pública da Escola de Enfermagem de Ribeirão Preto - USP.

2 Enfermeira. Doutoranda em Enfermagem de Saúde Pública. Dep. Enfermagem Materno Infantil e Saúde Pública.

${ }^{3}$ Enfermeira. Doutoranda em Enfermagem de Saúde Pública.

${ }^{4}$ Enfermeiro. Doutorando em Enfermagem de Saúde Pública. Prof. Fundação Educacional de Fernandópolis.

${ }^{5}$ Enfermeira. Doutoranda em Enfermagem de Saúde Pública. Prof. Fundação Educacional de São José do Rio Preto.
} 
A prática na vigilância...

\section{INTRODUÇÃO}

Os serviços de saúde num município devem ser organizados de modo a cumprir, simultaneamente, essas duas funções próprias: atender a demanda das pessoas, a partir de suas necessidades sentidas e enfrentar de forma programada, os problemas de saúde prioritários, a partir de um diagnóstico epidemiológico. Em outros termos, a oferta de serviços deve ser capaz de atender e resolver os problemas sentidos pelas pessoas e de combater os problemas mais importantes na coletividade. Essas duas tarefas implicam duas modalidades distintas de práticas em saúde, envolvendo tecnologias distintas, assim como lógicas diferenciadas de organização do trabalho em saúde. Há portanto, diferentes características nos processos de produção de ações para atender diferentes finalidades ; seja de prevenção e controle da doença na sociedade, seja de recuperação de doentes e estas práticas são realizadas por diferentes agentes que utilizam saberes que privilegiam mais a clínica ou a epidemiologia. A primeira tem na clínica o paradigma de conhecimento e organização da prática, essencialmente voltada para a atenção individual. A segunda baseia-se na Epidemiologia, como instrumento de conhecimento da realidade em saúde e agrega ao saber possibilitado pela clínica uma dimensão social e cultural indispensável à intervenção sobre o coletivo. Ambas as abordagens das lógicas de trabalho em saúde são importantes, além de complementares, dentro de uma visão mais compreensiva do processo saúde-doença (PAIM,1999).

A forma de organizar a atenção em saúde predominante na rede básica de serviços tem se norteado por programas e, baseia-se em protocolos de procedimentos e definição de ações de saúde centradas nos conhecimentos técnicos dos especialistas e profissionais de saúde.

As práticas de VE se estruturam através da lógica de programas de saúde, que são orientados conforme o modo sanitário de apreensão; possuem uma racionalidade sanitária que articula ações médicas e ações de saúde coletiva.

No debate que se coloca neste estudo, ainda que no plano conceitual, em torno da constituição do SUS, diz respeito às duas posicões que serão descritas em traços muito gerais e discutidas sob a ótica dos profissionais de saúde do nivel local e da vigilância epidemiológica em relação ao próprio conceito que se refere à prática de vigilância: generalidade versus especificidade, que se desdobram em duas possibilidades organizacionais: "conjunto indiferenciado de práticas e conjunto singular das práticas" (CAMPOS,1997, p.121).

A perspectiva de compreender as possibilidades de organização da prática de Vigilância Epidemiológica (VE) no sistema local de saúde, em nivel de atenção básica, vem acompanhada da necessidade de explicitar quais seriam as ações especificas do profissional de nivel local e quais seus limites de competência e de responsabilidade, e como organizar um serviço que vai atuar tanto sobre necessidades epidemiologicamente definidas (riscos epidemiológicos) quanto sobre a demanda por atenção?

A revisão critica do Sistema de Vigilância Epidemiológica ( SVE) produziu, basicamente, duas posições com relação ao próprio conceito de vigilância: uma aponta na direção da superação da dicotomia entre as chamadas práticas coletivas (Vigilância epidemiológica e sanitária) e as práticas individuais (assistência ambulatorial e hospitalar), através da elaboração do conceito de Vigilância à Saúde; outra, aponta para a ampliação do âmbito de atuação do SVE para além dos problemas relativos ao controle de doenças transmissiveis, retendo, entretanto, as especificidades quanto ao objeto e ao método de intervenção (BARATA, 1992).

Parece estar em jogo uma certa compreensão do trabalho em saúde que, embora não explicitada formalmente, informa as posições em conflito.

De um lado, a visão de um trabalho coletivo, relativamente indiferenciado no sentido de tomar o processo saúde-doença enquanto totalidade, como objeto para a ação, realizado através de atividades que também não percam de vista a concepção do processo saúde-doença como totalidade, porém composto por diferentes trabalhos especificos.

De outro lado, aponta para a ampliação do âmbito de atuação do SVE para além dos problemas relativos ao controle de doenças transmissiveis, retendo, entretanto, as especificidades quanto ao objeto e ao método de intervenção; ,embora assumindo a necessidade de ampliação do raio de ação dos Sistemas de Vigilância Epidemiológica, busca preservar certas características específicas desse conjunto de atividades. Assim são considerados objetos das práticas de vigilância aqueles problemas de saúde (doenças transmissiveis ou não, fatores de risco, etc...) que, por sua magnitude, transcendência, gravidade e vulnerabilidade e determinadas intervenções tecnológicas, mostrem-se adequados a pertencerem ao Sistema. Esta proposição, portanto, amplia o âmbito da vigilância epidemiológica, pela diversificação dos objetos-problema, sem descaracterizar um certo processo de trabalho que a distingue de outras práticas no interior dos serviços de saúde (programação, monitoramento, eventos-sentinela e etc.) (BARATA,1992).

\section{OBJETIVO}

Analisar as perspectivas de organização da prática de Vigilância Epidemiológica no sistema local de saúde sob a ótica dos profissionais de saúde do nível local e da VE.

\section{QUADRO TEÓRICO}

Campos (1997) sugere uma aplicação dos conceitos de campo e de núcleo o que contribui para pensar a prática na V.E. e suas especializações, que implicam recortes verticais no saber (área de competência) e na prática (área de responsabilidade). Núcleo é entendido como o conjunto de saberes e de responsabilidades específicos de cada profissão ou especialidade. O núcleo marca, dessa forma, a diferença entre os membros de uma equipe. Os conhecimentos e ações de exclusiva competência de cada profissão ou especialidade são os elementos de singularidade que definem a identidade de cada profissional ou especialista.

Campo de competência e de responsabilidade são os saberes e responsabilidades comuns ou confluentes à várias profissões ou especialidades, como por exemplo, todo o saber básico do processo saúde-doença. Seriam os conhecimentos sobre a relação profissional/paciente, sobre 
o risco epidemiológico e regras gerais de promoção e prevenção, bem como noções genéricas sobre política, organização de modelos e do processo de trabalho em saúde.

Nesta perspectiva, busca-se sinalizar a ampliação das responsabilidades dos serviços de saúde, incluindo aí um projeto terapêutico que articule lógicas diferenciadas de trabalho, tanto das equipes que atuam na assistência à demanda como nas equipes especializadas em saúde pública. Equipes que tanto agiriam diretamente sobre o território, de forma complementar, quanto também articulariam as ações de outros agentes, fossem eles de outros setores ou do próprio sistema de saúde.

Este estudo partiu dos seguintes pressupostos:

A Vigilância Epidemiológica, enquanto um conjunto de ações programáticas:

- É uma prática articulada à organização dos serviços de saúde. Se baseia em saberes estruturados histórica e socialmente, que envolvem a dimensão clinica, epidemiológica e gerencial. "Utiliza saberes especificos para controlar (evitar+tratar) a doença no plano coletivo". (MENDES GONÇALVES, 1992, p. 32 ).

- Possui uma área de competência (saber) e de prática (responsabilidade). A área de competência e de prática da VE não implica negar a existência de espaços especializados e não é especificidade de uma profissão.

\section{METODOLOGIA}

As atividades da Vigilância Epidemiológica (VE) na Secretaria Municipal da Saúde (SMS-RP) se distribuem em nivel central, distrital e local, e tem diferentes atribuições em cada nivel de atuação, totalizando 41 agentes distribuidos entre diferentes categorias, havendo o predominio de enfermeiras, médicas e auxiliares de enfermagem. A pesquisa teve aprovação do Comitê de Ëtica do Centro de Saúde Escola da Faculdade de Medicina de Ribeirão Preto-USP e dos agentes envolvidos da Secretaria Municipal de Saúde de Ribeirão Preto através de um termo de consentimento livre e esclarecido individual. Os dados foram coletados em 1998.

Através de uma abordagem qualitativa utilizou-se como instrumento de coleta de dados:

Fontes documentais: Relatóro da III Conferencia de Saúde do Município, Relatório do Departamento de VE- 1996, Relatório do I Seminário Integrado do Município(1996) e Plano de Saúde do Município (1998).

Entrevista semi-estruturada: As questões aplicadas para os dois grupos foram as seguintes: Quem deve responsabilizar-se pelas ações de Vigilância Epidemiológica? Qual é o preparo e disponibilidade das equipes de nivel local para realizar essas ações? Quem deve realizar as ações de VE? Que ações são realizadas e por quem?

O critério da escolha dos entrevistados, em número de 17 (dezessete), foi intencional, a partir do posto de trabalho que cada sujeito ocupava no sistema local de saúde. O grupo I é constituido por enfermeiras da VE da SMS-RP que trabalham nos cinco distritos de saúde e no nivel central da VE da SMS-RP, num total de 11 enfermeiras, sendo 5 enfermeiras que atuam no nivel central e 6 enfermeiras no nível distrital, escolhidas segundo os critérios: no mínimo uma de cada distrito, uma de cada programa no nível central e com maior tempo de trabalho na VE, identificado pela sigla (Ent. E $n^{\circ}$ ). O grupo II é composto por representantes dos gerente/gestores/ocupantes de cargo de direção de órgão de nivel central, distrital e local (assessoria de secretário da saúde - 1 representante; 1 diretor do planejamento em saúde; 1 diretor da Vigilância Epidemiológica; 3 gerentes de unidades de saúde); identificado pela sigla (Ent. $G n^{\circ}$ ).

Quanto à análise dos dados, buscou-se seguir os passos preconizados por Minayo, quando faz a discussão da análise de conteúdo, proposta por Bardin.

Uma das técnicas de análise de conteúdo é a análise temática onde busca-se descobrir os "núcleos de sentido que compõem uma comunicação cuja presença ou freqüência signifiquem alguma coisa para o objetivo analítico visado (...) a análise temática se encaminha para a contagem de freqüência das unidades de significação como definitórias do caráter do discurso" Minayo (1993, p.209).

Operacionalmente, a análise de conteúdo pode abranger as seguintes fases: pré-análise, exploração do material, tratamento dos resultados obtidos e interpretação, acompanhando o preconizado por Minayo (1993, p.210)."É na fase pré-analítica que se determinam as unidades de registro, a unidade de contexto (a delimitação do contexto de compreensão da unidade de registro), os recortes, a forma de categorização e os conceitos teóricos mais gerais que subsidiarão a análise".

Quanto à classificação dos dados, para o trabalho operacional de ordenação e separação dos aspectos de análise utilizou-se o software ETHNOGRAPH 4.0.

De acordo com as falas dos entrevistados, foram configurando-se várias unidades de registro agrupadas nos seguintes núcleos de sentido: novas formas de organizar o trabalho - articulação entre a equipe de VE e os niveis de assistência, trabalho articulado/ integrado; responsabilidade na execução das ações da VE - conhecimento específico e compromisso. Estes núcleos de sentido foram reagrupados para efeito de análise e interpretação e conformado em uma unidade temática eleita neste estudo: A Prática na Vigilância Epidemiológica: entre o geral e o específico.

Este tema é resultante da articulação entre o quadro teórico que norteou a investigação e os dados empíricos do trabalho de campo.

\section{RESULTADOS}

Esses depoimentos, a seguir, abrem a discussão da questão da descentralização das ações da VE para o território da UBS quanto às formas de organização do trabalho em saúde, que implicam considerar a V.E. tanto uma especialidade (conjunto singular das práticas de vigilância epidemiológica), quanto um corpo de saberes básico (conjunto indiferenciado de práticas de vigilância), para todas as profissões da saúde.

Eu acho que as ações da V.E. devem ser desenvolvidas a nível básico de atendimento. Ela pode estar a nível de distrital, com coordenação municipal. Eu não acredito num trabalho separado de V.E., que tem determinados profissionais, uma outra estrutura para realizar(...) (Ent. E.n¹). 
O depoimento ao mesmo tempo que propõe descentralizar as ações para serem executadas pela equipe de nivel local, não deixa claro qual seria o papel desta equipe e qual o apoio técnico da V.E. neste nivel.

Um dos aspectos que podem ser considerados, parte da premissa de que os profissionais da UBS tenham o conhecimento sobre o risco epidemiológico e as ações de controle da V.E., como o campo de saber básico do processo saúde-doença, além das ações burocráticas relacionadas ao fluxo de informação. Supõe-se também que, com a descentralização, os profissionais do atendimento iriam assumir a responsabilidade de desencadear as medidas de controle imediato do caso.

A abordagem da vigilância que predomina no depoimento da enfermeira $n^{\circ} 1$, se desdobra em termos organizacionais, em um conjunto indiferenciado de práticas, e tende reforçar os niveis locais como concentradores de decisões e recursos atribuindo ao profissional da UBS a execução das ações de V.E. quando está prestando o atendimento clínico ao paciente, o que reduz as atividades de prevenção e cura ao mesmo processo de trabalho

Existe uma proposta de que a V.E. chegue até a UBS. A área de abrangência é muito menor. Se a unidade absorvesse a V.E. a ação dela seria muito mais rápida. Cada unidade deveria se conscientizar que deve fazer vigilância em sua área de abrangência(...) (Ent. E.n9).

A justificativa para a descentralização da V.E. está relacionada ao processo de conscientização da equipe de saúde da UBS para assumir atribuições da V.E., acompanhada de uma proposta de capacitação de pessoal e de mudança de lógica de assistência que envolve um preparo para desenvolver esse trabalho.

Eu acho pouco o número de profissionais na V.E. Necessitava-se de muito mais gente (Ent. E.n`9).

Quero dizer que nós temos mais de 30 unidades básicas de saúde (UBS) e a enfermeira ou o médico que trabalham na unidade não sabem que no quarteirão do lado tem uma pessoa com tuberculose. E o primeiro passo, é capacitar as equipes da UBS para a prevenção, controle de comunicantes, técnicas e instrumentos de avaliação. (Ent. G.n²).

O depoimento do gestor considera os instrumentos e saberes da V.E. que auxiliam na avaliação das ações da própria V.E. e da Unidade como um todo porque o serviço local está organizado para prestar assistência ao doente. Ressalta a necessidade de articulação das ações de saúde dentro da própria UBS, entre os setores que prestam assistência, e os condicionantes de ordem politica que interferem no processo.

Não se constrói a descentralização da V.E. em quatro anos de governo. As rupturas são problemáticas. As pessoas são escolhidas por critérios políticos ( ). O desafio é parte de um projeto global. Falta uma política de governo do município. Não adianta serviços separados, que não estão articulados ao plano de saúde do município. Tudo é um processo e leva tempo (Ent. G.n¹).

Há porém, um desconhecimento por parte da equipe da UBS, das ações e informações epidemiológicas no sentido de desencadear a prevenção, controle e avaliação, o que requer especificidade do campo de trabalho.

A V.E. é um serviço de nível secundário e não tem espaço para ser realizado em uma U.B.S., pois o nome já fala: Unidade BÁSICA, e nem tem espaço físico aqui para a V.E., não tem espaço nem para o atendimento. (Ent. G.n4).

A gente trabalha única e exclusivamente para a vigilância. Precisamos envolver a unidade nisso tudo para não ficar algo isolado só da vigilância, a qual deve dar respaldo técnico. (Ent. E. n8).

Há um desejo de preservar a especificidade do trabalho da V.E., seu espaço e equipe, o que tem reforçado a distância entre as equipes, seja as da UBS, seja as da V. E.

Questão da V.E. é para a V.E. do Distrito (...), que isso é problema deles. Porque o que nós podemos oferecer é consulta médica. (Ent. G.n4).

Na relação de trabalho entre as Unidades Básicas de Saúde e a Vigilância Epidemiológica há desconhecimento, falta de preparo e de compromisso dos profissionais de saúde da unidade, que muitas vezes não estão atentos às doenças de notificação compulsória, além das dificuldades na abordagem de casos pela unidade. (Ent. E.n4).

Nós temos hoje muitas unidades sem notificar(...),

Com isto, investe-se em treinamento de funcionários, realizado pelas enfermeiras da Vigilância Epidemiológica, do nivel Distrital, para que estes funcionários conheçam as normas e a área de atuação.

A proposta da UBS é atender PA e não estabelece relações com sintomas sugestivos de DNC como por exemplo a Tuberculose, que necessita de uma investigação melhor, tem que haver um envolvimento, compromisso com o que você está fazendo (Ent. E. $\left.n^{\circ} 6\right)$.

Esses depoimentos mostram uma separação muito nítida da prática na UBS e na V.E. A prática na UBS, que oferece o atendimento médico evidenciando o modelo do Pronto Atendimento, sem a preocupação maior com a busca ativa de casos, com o diagnóstico precoce, com ações terapêuticas e educativas de promoção da saúde e prevenção de doenças. E a Vigilância Epidemiológica atua nos moldes verticalizados dos programas de saúde pública. Ambas têm finalidades distintas, utilizam instrumentos e agentes diferentes para operá-las em espaços diversos, acarretando, na prática, um afastamento entre os agentes.

As atribuições especificas dessas equipes de V.E., segundo Campos (1997) seria a de realizar de forma sistemática e planejada diagnósticos de mortalidade e morbidade, avaliação da eficácia dos serviços e dos graus de riscos dos vários agrupamentos populacionais, dirigir e coordenar ações específicas voltadas para o controle ou erradicação de doenças de massa e dar apoio a todos que trabalhem o tema da formação de uma nova consciência sanitária entre os trabalhadores de saúde e a população.

As abordagens em relação às possibilidades de organização das práticas de vigilância, apresentadas por Campos(1997) e Barata (1992), respectivamente, encaminham para a questão sobre considerar a saúde pública e a V.E. uma especialidade (conjunto singular das práticas de vigilância epidemiológica), e defendem a 
existência de equipes de saúde pública para atuar como apoio às equipes das unidades de saúde em nivel local.

Campos (1997) sugere que as ações de saúde pública, como a Vigilância Epidemiológica, dentre outras, deveriam ser divididas entre a rede básica e os núcleos de saúde coletiva em cada distrito de saúde. Ou seja, mesmo quando as equipes locais interviessem diretamente, deveriam ser apoiadas por grupos de sanitaristas. Algumas medidas como por exemplo, coordenação de campanhas para o controle da dengue ou investigação de um agravo súbito e inesperado, deveriam estar a cargo destes sanitaristas que utilizam, prioritariamente, o enfoque epidemiológico para orientar sua prática para o campo da prevenção. Tal prática implica especificidade de conhecimentos e ações de exclusiva competência da saúde pública, ou seja, os elementos de singularidade que definem a identidade desta especialidade.

Nessa abordagem, a equipe de saúde pública justifica-se pelo fato de que o sistema local deve assumir, progressivamente, a responsabilidade pela implementação de grande parte das ações de impacto coletivo, sem o que nunca se atingiria a integração sanitária.

Este conhecimento especifico tem conferido à equipe de VE possibilidade de planejar, realizar levantamentos para o diagnóstico de saúde, estabelecer prioridades, executar e avaliar perfis epidemiológicos e situações de saúde, conforme os depoimentos a seguir.

Mas, também, temos uma capacidade de atuar mais de imediato num fato que acontece, se há um risco de agravo à saúde, detectamos esse fato e levamos propostas para a chefia e para o secretário (Ent. E. $n^{\circ} 3$ ).

Tem que ter equipes específicas de V.E. para trabalhar a informação, dar retorno para as equipes da UBS(...) (Ent. G.n¹).

A forma de organização do trabalho da V.E. em um território apresenta possibilidades de construção de diferentes graus de autonomia, uma vez que ela não está diretamente subordinada ao projeto adotado para a produção de cuidados individuais de diagnóstico e terapêutico.

Segundo Campos (1997) um dos principais segredos para assegurar a qualidade em saúde estaria na adequada combinação de autonomia profissional com certo grau de definição de responsabilidade para os trabalhadores. (...)A autonomia-responsável somente aconteceria quando houvesse interesse e envolvimento dos agentes com uma certa tarefa.

\section{CONSIDERAÇÕES FINAIS}

Há um desejo de preservar a especificidade do trabalho da V.E., seu espaço e equipe, o que tem reforçado a distância entre as práticas de saúde, seja as de âmbito individual (Pronto-Atendimento nas UBS), seja as de alcance coletivo.

Na relação de trabalho entre as Unidades Básicas de Saúde e a Vigilância Epidemiológica há desconhecimento e falta de preparo e das equipes locais, que deveriam ser apoiadas por grupos da VE do distrito.

A prática da V.E. aponta potencialidades que implicam em maiores responsabilidades para a equipe no nivel local que acarretam definições como: área de abrangência dos serviços, perfil demográfico e sócioeconômico da população atendida, conjunto de equipamentos e instalações existentes, bem como um número de profissionais preparados para atuaremum modelo de saúde que organiza intervenções na perspectiva da construção de uma prática de vigilância à saúde.

A descentralização deve vir acompanhada de uma mudança no enfoque da atenção, que além do cuidado ao doente, no âmbito individual, se volte para o gerenciamento e controle das ações de saúde que incidem sobre o seu território, entendido no âmbito coletivo, o que exige qualificação técnica dos profissionais e agentes envolvidos acompanhada de conhecimento técnico e responsabilidade das ações para o nivel da UBS. Tais práticas apontam a possibilidade de incorporação de outros sujeitos, gerentes de serviços, técnicos e representantes de grupos organizados da população. A equipe de $V$. $E$ tem reforçado a necessidade de encontrar um interlocutor nas Unidades, que se responsabilize pelas ações de V. E.

\section{REFERÊNCIAS BIBLIOGRÁFICAS}

BARATA, R. Reorientação das práticas de vigilância epidemiológica. In: SEMINÁRIO NACIONAL DE VIGILÂNCIA EPIDEMIOLÓGICA,1992, Brasília. Anais... Brasília: MS/FNS/ CENEPI/UVE, 1992.

CAMPOS, G.W.S. Análise crítica das contribuições da saúde coletiva e a organização das práticas de saúde no SUS. In: FLEURY, S. (Org.) Saúde e democracia: a luta do CEBES. São Paulo: Lemos Editorial, 1997. p. 113-124.

MENDES GONÇALVES, R. B. Práticas de saúde: processos de trabalho e necessidades. Cadernos CEFOR, 1992. 54 p.

MINAYO, M.C.F. O Desafio do Conhecimento: pesquisa qualitativa em saúde. 2. ed. São Paulo: HUCITEC/ABRASCO, 1993. 269 p.

PAIM, J.S. Políticas de descentralização e atenção primária à saúde. In: ROUQUAYROL,M.Z. Epidemiologia \& Saúde. São Paulo: MEDSI, 1999. cap. 18, p. 455-466. 